\title{
Relative importance of clinical and sociodemographic factors in association with post-operative in-hospital deaths in colorectal cancer patients in New South Wales: An artificial neural network approach
}

\section{Sha Sha MPH ID | Wei Du PhD ID | Anne Parkinson PhD | Nicholas Glasgow MBChB, MD}

Research School of Population Health, Australian National University, Canberra, Australia

\section{Correspondence}

Sha Sha, Research School of Population Health, Australian National University, Building 63, Eggleston Road, Canberra, ACT 2601, Australia.

Email: Sha.Sha1119@hotmail.com

\begin{abstract}
Rationale, Aims and Objectives: Co-morbidities in colorectal cancer patients complicate hospital care, and their relative importance to post-operative deaths is largely unknown. This study was conducted to examine a range of clinical and sociodemographic factors in relation to post-operative in-hospital deaths in colorectal cancer patients and identify whether these contributions would vary by severity of co-morbidities.
\end{abstract}

Methods: In this multicentre retrospective cohort study, we used the complete census of New South Wales inpatient data to select colorectal cancer patients admitted to public hospitals for acute surgical care, who underwent procedures on the digestive system during the period of July 2001 to June 2014. The primary outcome was inhospital death at the end of acute care. Multilayer perceptron and back-propagation artificial neural networks (ANNs) were used to quantify the relative importance of a wide range of clinical and sociodemographic factors in relation to post-operative deaths, stratified by severity of co-morbidities based on Charlson co-morbidity index. Results: Of 6288 colorectal cancer patients, approximately 58.3\% $(n=3669)$ had moderate to severe co-morbidities. A total of $464(7.4 \%)$ died in hospitals. The performance for ANN models was superior to logistic models. Co-morbid musculoskeletal and mental disorders, adverse events in health care, and socio-economic factors including rural residence and private insurance status contributed to post-operative deaths in hospitals.

Conclusion: Identification of relative importance of factors contributing to inhospital deaths in colorectal cancer patients using ANN may help to enhance patient-centred strategies to meet complex needs during acute surgical care and prevent post-operative in-hospital deaths.

\section{KEYWORDS}

artificial neural network, colorectal cancer, co-morbidities, in-hospital mortality, patient-centred strategy, post-operative care 


\section{1 | INTRODUCTION}

Colorectal cancer (CRC) is a global population health burden on health care systems with an estimated $60 \%$ increase in new cases and 1.1 million deaths by $2030 .{ }^{1}$ In Australia, it was the third most commonly diagnosed malignancy and the second leading cause of cancer-related death. ${ }^{2}$ Despite the fact that CRC outcomes have improved dramatically in the past few decades, including improved survival, ${ }^{3}$ a continuing focus on improvement in cancer-related outcomes remains a population health priority. ${ }^{4}$

Co-morbid conditions are frequently seen in CRC patients. ${ }^{5}$ In such patients, acute surgical care outcomes may be worse compared with patients without co-morbidities, ${ }^{6,7}$ including increased postoperative mortality rates. ${ }^{8-10}$ Given that co-morbid conditions are highly correlated with many sociodemographic factors, ${ }^{11}$ an understanding of the relative contribution of co-morbid conditions and sociodemographic characteristics to post-operative mortality during acute inpatient care may help to improve both clinical practice and patient outcomes. ${ }^{12}$

Previous studies based on generalized linear regression models were developed to predict post-operative outcomes and explore the association with co-morbidities. ${ }^{7,10}$ However, few such models have attempted to address the impact of each co-morbid condition on deaths during acute inpatient care, especially when these comorbidities may be highly correlated with other sociodemographic characteristics. ${ }^{11}$ Given that the use of generalized linear regression to model highly correlated data could potentially result in incorrect identification of exposure factors in relation to outcomes, ${ }^{13}$ the application of artificial neural networks (ANNs) offers more flexible algorithms that outperform the conventional generalized linear regression models in terms of discrimination, calibration, and accuracy when dealing with complex scenarios. ${ }^{14}$ ANNs attempt to mimic the way human brains work to provide the ability to develop new information and have been proposed for clinical outcome prediction in CRC patients ${ }^{15}$ However, the application of ANNs in evaluating a range of highly correlated factors in relation to poor post-operative outcomes during acute hospital care is less common compared with the use of conventional models.

Using the ANN modelling approach, this study examined to what extent various clinical and sociodemographic factors in CRC patients might be responsible for in-hospital post-operative deaths and which of these factors became relatively more important as co-morbidity severity increased. Findings may inform strategies to improve acute surgical care and prevent post-operative in-hospitals death.

\section{2 | PATIENTS AND METHODS}

\section{1 | Data source}

We employed the NSW Admitted Patient Data Collection (APDC), a complete census of hospital episodes of care for admitted patients maintained by the New South Wales (NSW) Department of Health, covering all public, private, and repatriation hospitals, and private day procedure centres in NSW, the most populous state in Australia. Although inclusion of private hospital data would most accurately measure possible variation in post-operative outcomes between public and private sectors, identifiers were not available for private hospital; hence, we restricted the current analysis to that of public hospitals. Medical reasons for hospital admission were coded at the time of disposition using the Australian modification of ICD-10 (ICD-10-AM). In accordance with the data use agreement (NSW Health Department), for each de-identified episodes of care, the following data were extracted: age, sex, marital status, postcode of usual residence, private insurance, urgency of admission, hours in intensive care (if applicable), up to 53 medical diagnoses, time to procedure, and disposition status. Ethics approval was obtained from the Institution Science and Medical Delegated Ethics Review Committee (\#2016/030).

\section{2 | Study setting}

We selected CRC patients age 45 years and older using ICD-10-AM codes, ie, C18 (malignant neoplasm of colon), C19 (malignant neoplasm of rectosigmoid junction), and C20 (malignant neoplasm of the rectum), who were admitted for acute hospital care and underwent a primary surgical resection for CRC, using ICD-10 procedure codes. ${ }^{16}$ We categorized all hospitalizations based on the reason for admission as either cancer diagnosis per se or others (eg, related gastrointestinal symptoms). We also categorized secondary diagnoses into major groups according to ICD-10-AM codes as co-morbid conditions (ie, D5-D9: blood immune systems; E: endocrine system; F: mental and behavioural disorders; I: circulatory system; J: respiratory system; K: digestive system; $\mathrm{M}$ : musculoskeletal disorders; $\mathrm{N}$ : genitourinary system). We further identified hospital acquired infections as well as complications based on the codes of Classification of Hospital Acquired Diagnoses $(\mathrm{CHADx})^{17}$ and categorized them as either yes or no. Each hospital was treated as an individual entity. We considered financial year as continuous, as well as time to surgery in days with any waiting period between hospital admission and the first surgery longer than 30 days collapsed into 31 days. We further categorized age in year groups (ie, 45-64, 65-84, or $85+$ ); sex as male or female; private insurance as yes or no; rurality of residence as urban or rural; socioeconomic status as first (most disadvantaged), second, third, fourth, or fifth (least disadvantaged) quintile using the postcode-based "Index of Relative Socio-Economic Disadvantage" (IRSD); ${ }^{18}$ intensive care (ICU admission) as yes or no; disposition status as death or alive at the end of acute care episode; hospital characteristics as principal referral, or other based on the Australian Institute of Health and Welfare classification system. ${ }^{19}$ A separate category was included for unclassifiable values.

Although 30-day post procedure mortality has been used as an endpoint in association with hospital care, this measure relies on linkage of hospitalization and death registry data using a unique personal identifier, which was unavailable in the current APDC data. While inhospital mortality has been used in a similar setting ${ }^{20}$ and 
demonstrated a substantially high agreement (Kappa > 80\%) with 30day mortality, ${ }^{21}$ it can be readily derived from de-identified hospitalization data and serve as a screening performance indicator for potential improvement in hospital care. ${ }^{22}$ In the current setting, in-hospital mortality was selected as our study outcome.

\section{3 | Artificial neural networks}

ANNs are inspired by biological neural networks, share a common feature of an interconnected group of nodes in multiple layers, and demonstrate an advantage in capturing highly complex (eg, nonlinear and collinear) patterns of input variables, as well as detecting all potential interrelationships between input variables. ${ }^{15}$ This study used the three-layer perceptron (MLP) back-propagation (BP) neural network, a popular supervised network architecture with robust performance for better prediction and classification in comparison with the most conventional model (logistic regression). Considering connection weights between neurons reflect the contribution of explanatory variables in relation to a response variable. ${ }^{23}$ We used the connection weight approach (CWA), which is suggested to be with a better performance than other approaches to ascertain the relative importance of each variable modelled in the $\mathrm{ANN},{ }^{23,24}$ by calculating the outcome of raw interlayer (ie, input-hidden and hidden-output) connection weights between neurons in each layer and then summing all the product of connection weights. ${ }^{24}$ CRC patients in this study were stratified into two groups based on the Charlson co-morbidity index (CCl) score with weight of 1 for co-morbid conditions such as chronic pulmonary disease; weight of 2 for co-morbid conditions such as hemiplegia or paraplegia; weight of 4 for co-morbid conditions such as moderate or severe liver disease; and weight of 6 for co-morbid other type of cancer. ${ }^{25}$ Given that $\mathrm{CCl}$ is a strong predictor for cancer mortality ${ }^{25}$ and has been validated by age-sex adjusted models, ${ }^{26}$ we applied the algorithm using ICD-10 codes from the studied hospitalization only (without a look back period) to only non-CRC diagnoses and did not consider age as a factor in calculating the $\mathrm{CCl}$. We categorized $\mathrm{CRC}$ patient with total $\mathrm{CCl}$ score $>2$ as moderate to severe comorbidity group and the rest as less severe group. ${ }^{27}$ To further enhance the stability of the models, we repeated 50 runs of ANN modelling for our sampled CRC patients in each group of co-morbidity severity, respectively, and calculated the average of model-derived relative importance estimates of each explanatory variable to identify factors with relatively strong effects on model outcome. ${ }^{28}$

To identify the number of hidden neurons with "optimal" performance, that is, having a lower misclassification error for the test data set, as well as preventing the "overfitting" of neural network, a 10-fold cross-validation approach was carried out. During each test, data were randomly split into 10 groups, nine groups were classified as training data set and the remaining group was treated as testing data set for each cycle in the validation. Before ANN model training, we used the combination of over-and-under sampling approach (the "ROSE" package in R.3.5.1) to create a possibly balanced distribution in dependent variable (ie, death) in our training sample sets, to improve the accuracy and reduce bias towards the majority class due to the unbalanced classification problem. ${ }^{29}$ The average misclassification error for each testing data set was calculated after implementing 10 cycles in each model. The cross-validation was started from using a small number of neuron nodes in the hidden layer and increased the number gradually until the test error started to increase.

We developed two MLP BP neural networks that consisted of one input layer, one hidden layer, and one output layer, ie, (model 1: for CRC patients with less severe comorbidities and model 2: those with moderate to severe co-morbidities), to identify the relative importance of explanatory variables in contribution to death. The input layer for each model comprised six sociodemographic and six hospital-related factors and 10 major co-morbidity groups, and the output layer comprised whether an in-hospital death has occurred or not. We normalized the data value for each explanatory variable using the formula $X_{i}=\left(X_{i}-X_{\min }\right) /\left(X_{\max }-X_{\min }\right)$ before building the models.

\section{4 | Statistical analysis}

Numbers and proportions were calculated for each variable. We examined correlation between predictive variables prior to modelling. MLP resilient BP ANN models with backtracking for classification problem were developed for patients with different severity of co-morbidities after identifying the "optimal" number of hidden nodes in the crossvalidation. We used the error function of cross entropy, and the activation function of "logistic sigmoid" in the hidden layer, and hyperbolic tangent in the output layer for the binary classification situation. Data were randomly split into training and testing data with the ratio of 7:3. The training data sets were used to train the ANN model and then putting the test data set to validate the predictive accuracy of the model. CWA was scaled between 0 and 10 , by extrapolating the standardization $X_{i-s c a l e}=X_{i}^{*} 10 /\left(X_{\max }-X_{\min }\right)$. We used the absolute value to rank the importance of variables but maintain the signs to identify their positive or negative influence on the outcomes. The larger a factor's CWA is, the more important is its contribution towards the study outcome. ${ }^{24}$

Additionally, we developed binary logistic regression models for each co-morbidity severity group using identical ANN model input. A link test was performed with a $P$ value of $<.05$ representing statistically significant specification errors for logistic regression models. The accuracy for confusion matrix (with the cut-off point of 0.5 ), area under curve (AUC), and Akaike information criteria (AIC) ${ }^{30}$ were compared between logistic regression models and ANN models. The statistical software R.3.5.1 ("neuralnet") was used.

\section{3 | RESULTS}

During the 13-year study period, a total of 6288 CRC patients who underwent a primary surgical resection for CRC were considered for the analysis. More than half of the study population $(58.3 \%, \mathrm{n}=$ 3669) had moderate to severe co-morbidities, and number of inhospital deaths increased with the increasing severity of co-morbidity (Table 1). The majority of the study population were aged 65 to 84 
years $(59.8 \%, n=3762)$ and admitted to principal referral hospitals for cancer-related acute care $(80.4 \%, n=5055)$. Digestive disorders was the most common co-morbid category in the study population (64.4\%, $\mathrm{n}=4049)$, followed by circulatory $(49.0 \%, \mathrm{n}=3080)$ and endocrine conditions (44.7\%, $\mathrm{n}=2813$ ).

\section{1 | Artificial neural networks}

Two MLP BP neural networks with the "optimal" neural structure were developed after cross-validation (Figure 1), with one bias neuron in each input layer and hidden layer, respectively. The overall

TABLE 1 Sociodemographic and clinical clinical characteristics by severity of comorbidities, number (\%)

\begin{tabular}{|c|c|c|c|}
\hline & Less Severe & Moderate to Severe & Total \\
\hline & No. (\%) & No. (\%) & No. (\%) \\
\hline Total & 2619 & 3669 & 6288 \\
\hline \multicolumn{4}{|l|}{ Sociodemographic characteristics } \\
\hline \multicolumn{4}{|l|}{ Sex } \\
\hline Males & $1253(47.8)$ & $1889(47.8)$ & $3152(50.1)$ \\
\hline \multicolumn{4}{|l|}{ Age groups } \\
\hline $45-64$ & $522(19.9)$ & $986(26.9)$ & $1508(24.0)$ \\
\hline $65-84$ & $1578(60.3)$ & $2184(59.5)$ & $3762(59.8)$ \\
\hline 2nd & $543(20.7)$ & $844(23.0)$ & $1387(22.1)$ \\
\hline $3 r d$ & $603(23.0)$ & $772(21.0)$ & $1375(21.9)$ \\
\hline 4 th & $471(18.0)$ & $673(18.3)$ & $1144(18.2)$ \\
\hline 5th (least) & $316(12.1)$ & $507(13.8)$ & $823(13.1)$ \\
\hline Being single & $1288(49.2)$ & $1662(45.3)$ & $2950(46.9)$ \\
\hline Having private insurance & $559(21.3)$ & $807(22.0)$ & $1366(21.7)$ \\
\hline Rural residence & $244(9.3)$ & $265(7.2)$ & 509 (8.1) \\
\hline \multicolumn{4}{|l|}{ Clinical characteristics } \\
\hline \multicolumn{4}{|l|}{ Primary reason for admission } \\
\hline Median (interquartile range) & $1(5)$ & $1(5)$ & $1(5)$ \\
\hline \multicolumn{4}{|l|}{ Comorbid conditions } \\
\hline Infections & $613(23.4)$ & $856(23.3)$ & $1469(23.4)$ \\
\hline Blood immune & 794 (30.3) & $1096(29.9)$ & $1890(30.1)$ \\
\hline Endocrine & 1089 (41.6) & $1724(47.0)$ & $2813(44.7)$ \\
\hline Mental & 305 (11.6) & 508 (13.8) & 813 (12.9) \\
\hline Circulatory & 1219 (46.5) & $1861(50.7)$ & $3080(49.0)$ \\
\hline Respiratory & $563(21.5)$ & 859 (23.4) & $1422(22.6)$ \\
\hline Digestive & 1705 (65.1) & 2344 (63.9) & $4049(64.4)$ \\
\hline Musculoskeletal & $156(6.0)$ & $270(7.4)$ & $426(6.8)$ \\
\hline Genitourinary & 497 (19.0) & 915 (24.9) & $1412(22.5)$ \\
\hline Complications & 521 (19.9) & $716(19.5)$ & $1237(19.7)$ \\
\hline
\end{tabular}




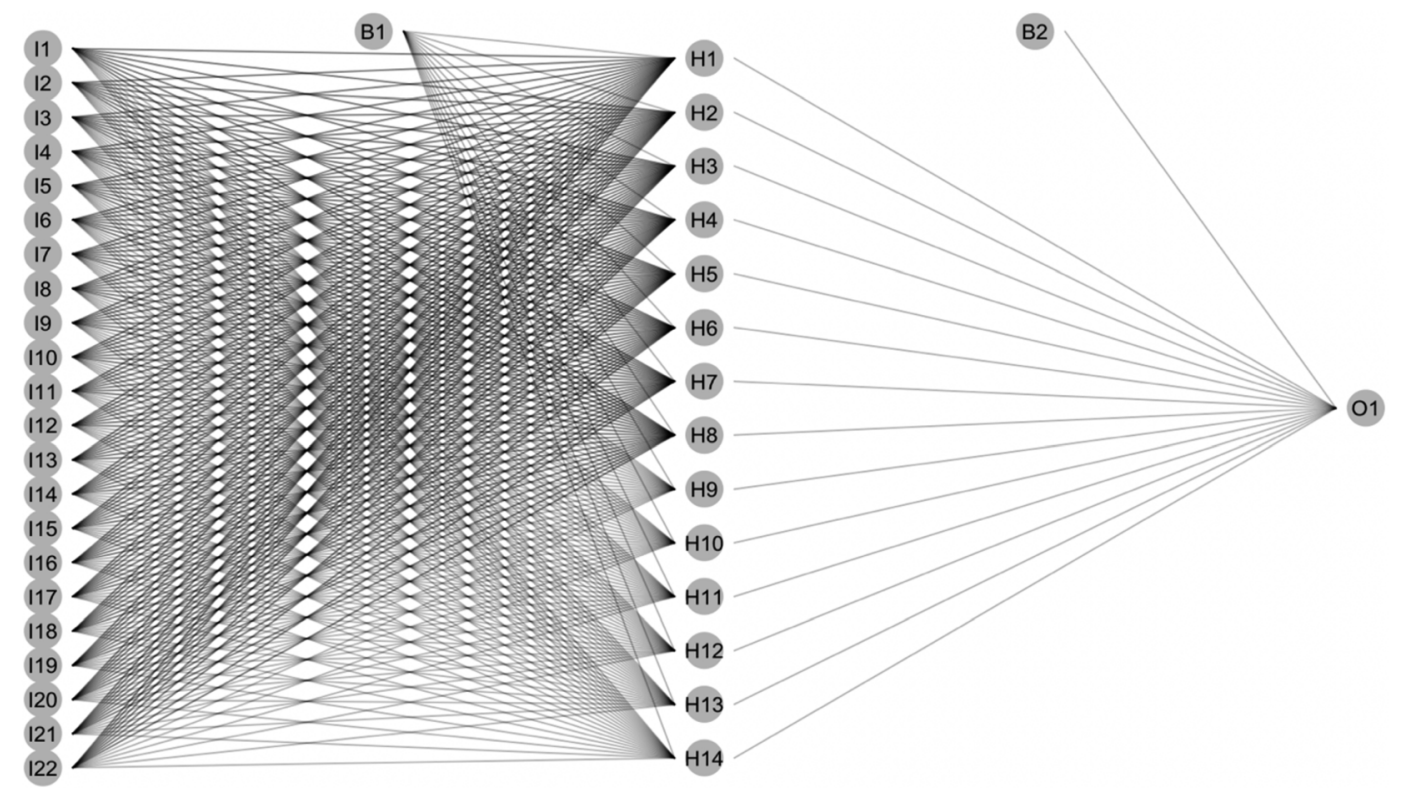

Input layer

Hidden layer

Output layer

FIGURE 1 Schematic diagram of the three 3-layer artificial neural network (ANN) models with 22 input nodes, 14 hidden nodes, and one output node

performance of ANN models across the two co-morbidity severity groups was superior to that of logistic models in terms of AUC and AIC (Table 2), capturing high-order interactions, non-linear relationships, and multicorrelation between input variables in the current setting. Logistic regression models were fitted with identical inputs, which failed the link test for model specification $(P<.0001)$, and therefore, the effect estimation for each input variable was not presented.

Figure 2 and Table 3 present the relative importance of predictors of in-hospital death for each ANN model corresponding to different co-morbidity severity groups. Leading factors in relation to post-operative deaths were similar across different co-morbidity severity groups, but with different ranking in importance. The presence of co-morbid musculoskeletal disorders, rural residence, private insurance, and infections were relatively more important than the other sociodemographic, hospital, and clinical factors as contributing towards post-operative deaths among CRC patients with less severe co-morbidities. For CRC patients with moderate to severe comorbidities, rural residence and infections were relatively more important, followed by co-morbid musculoskeletal and mental disorders. For this group, a number of factors appeared to be protective for in-patient death including less disadvantaged socio-economic status, operations in recent years, if the admission was primarily for cancer care, and having co-morbid digestive disorders. Being female was associated with a lower risk of post-operative death in the group with less severity. In the group with moderate to severe co-morbidities, principal hospital admission and holding private health insurance were positively associated with post-operative inhospital survivals.

\section{4 | DISCUSSION}

To the best of our knowledge, this is the first study of its kind to employ a neural network for quantifying relative importance of a wide range of predictors in relation to post-operative in-hospital death in CRC patients using a state-wide population-based inpatient data collection. Given the wealth of clinical and sociodemographic inputs in relation to post-operative deaths at the end of acute care, conventional logistic regression models with identical inputs experience severe misspecification errors, implying a high risk of identifying the wrong predictors due to model misspecification. ${ }^{13}$ Compared with more conventional logistic models, ANN models have demonstrated better predictive power (larger AUC) and model fit (smaller AIC)

TABLE 2 Model performance of ANN vs. logistic regression

\begin{tabular}{|c|c|c|c|c|c|c|}
\hline \multicolumn{4}{|l|}{ Less Severe } & \multicolumn{3}{|c|}{ Moderate to Severe } \\
\hline Models & AUC & Accuracy & AIC & AUC & Accuracy & AIC \\
\hline Logistic regression & 0.88 & 0.95 & 846.0 & 0.84 & 0.91 & 1752.8 \\
\hline
\end{tabular}


(A) Relative Importance of Variables for the Less Severe Group with Death Outcome (ANN MODEL SET 1)

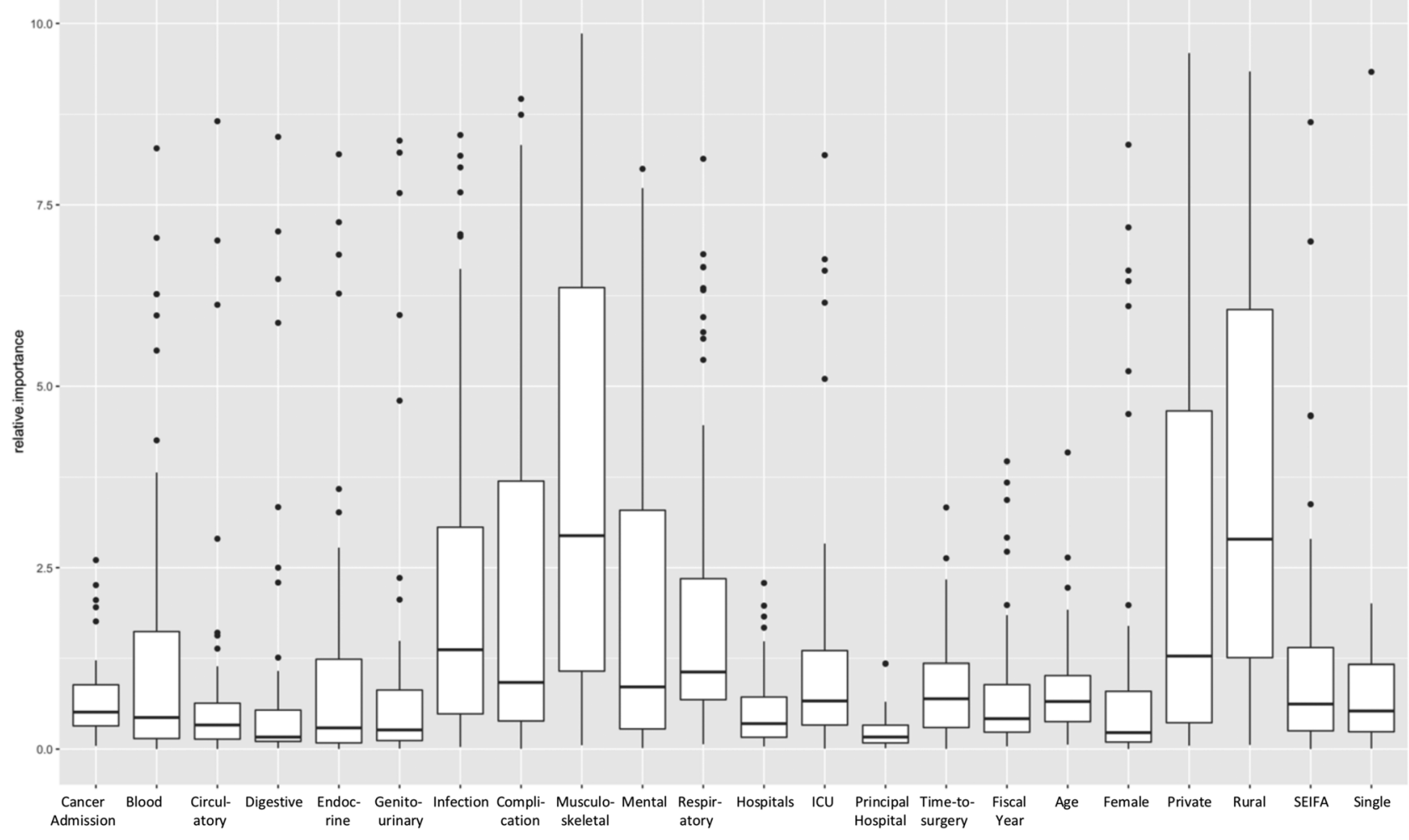

(B) Relative Importance of Variables for the Moderate to Severe Group with Death Outcome (ANN MODEL SET 2)

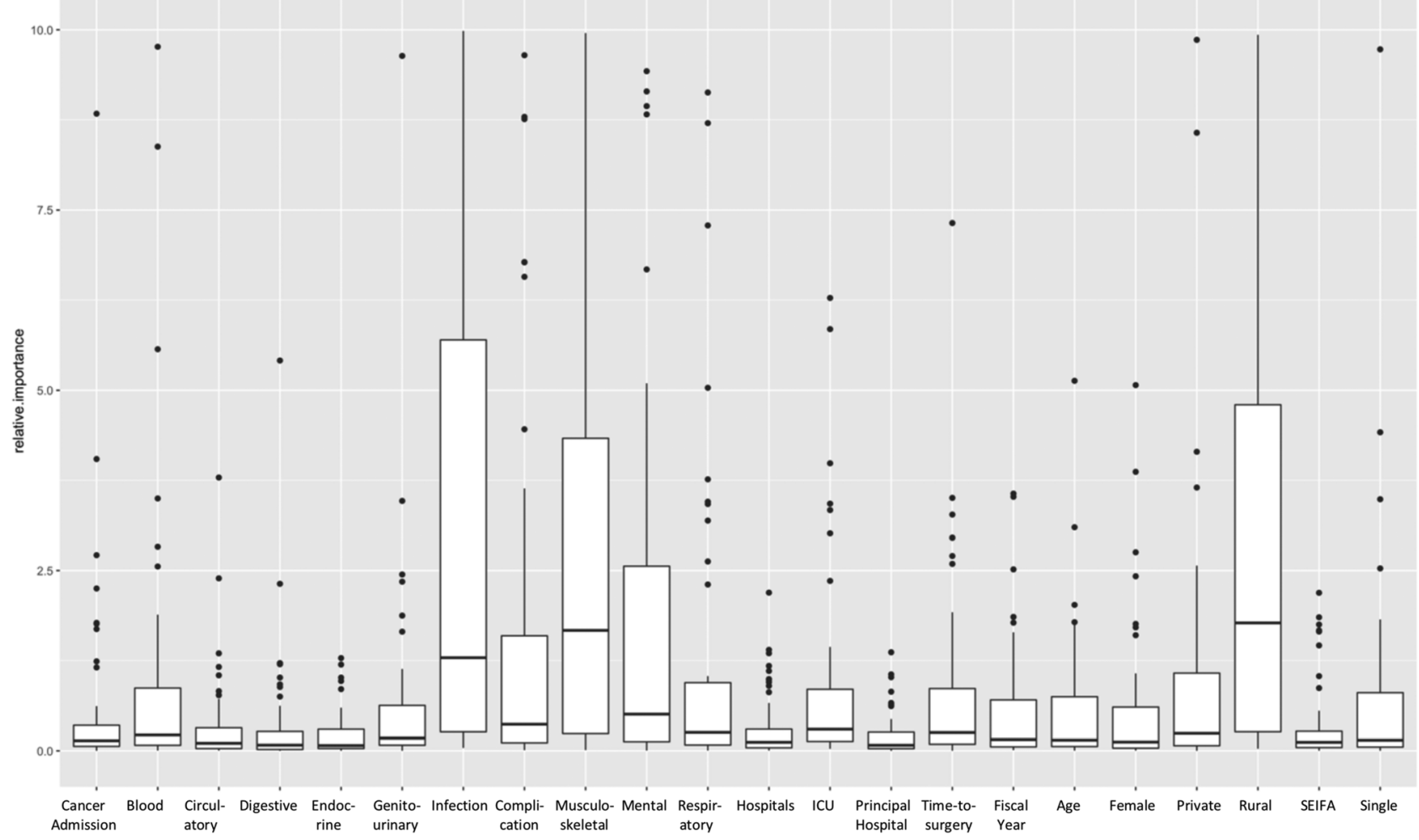

FIGURE 2 Absolute relative importance of factors in relation to in-hospital mortality in colorectal cancer patients. (A) Patients with less severe comorbidities and (B) patients with moderate to severe comorbidities. Note: SEIFA: socio-economic index (from the most disadvantage to the least disadvantage). [Correction added on 4 December 2019, after first online publication: In Figure 2, a note has been added to the legend in this current version.] 
TABLE 3 Relative importance of factors in relation to in-hospital deaths

\begin{tabular}{|c|c|c|c|}
\hline $\begin{array}{l}\text { ANN Model } 1 \text { - - } \\
\text { Less Severe (Death) }\end{array}$ & $\begin{array}{l}\text { ANN Model } 2 \text { - - } \\
\text { Moderate to Severe } \\
\text { (Death) }\end{array}$ & & \\
\hline M1 factor & AMI & M2 factor & AMI \\
\hline Musculoskeletal & 3.81 & Rural & 3.13 \\
\hline Rural & 3.68 & Infections & 2.93 \\
\hline Private Insurance & 2.72 & Musculoskeletal & 2.76 \\
\hline Complication & 2.29 & Mental & 1.85 \\
\hline Infections & 2.26 & Complication & 1.64 \\
\hline Mental & 2.18 & Blood-Immune & 1.22 \\
\hline Respiratory & 2.17 & Private Insurance $^{* a}$ & 1.04 \\
\hline Blood-Immune & 1.53 & Respiratory & 0.99 \\
\hline ICU & 1.37 & ICU & 0.88 \\
\hline $\begin{array}{l}\text { Socioeconomic } \\
\text { status }^{\text {a* }}\end{array}$ & 1.32 & Time-to-surgery & 0.76 \\
\hline Female $^{a *}$ & 1.19 & Single & 0.71 \\
\hline Endocrine & 1.15 & Genitourinary & 0.67 \\
\hline Genitourinary & 1.10 & Cancer admission $^{* a}$ & 0.65 \\
\hline Digestive $^{\mathrm{a} *}$ & 0.96 & Age group & 0.57 \\
\hline Time-to-surgery & 0.90 & Female & 0.57 \\
\hline Circulatory & 0.88 & Fiscal year ${ }^{* a}$ & 0.54 \\
\hline Single & 0.85 & Digestive $^{* a}$ & 0.37 \\
\hline Age group & 0.85 & $\begin{array}{l}\text { Socioeconomic } \\
\text { status }^{*}{ }^{* a}\end{array}$ & 0.36 \\
\hline Fiscal year ${ }^{a *}$ & 0.82 & Circulatory & 0.36 \\
\hline Cancer admission ${ }^{\mathrm{a} *}$ & 0.64 & Hospitals & 0.33 \\
\hline Hospitals & 0.57 & Endocrine & 0.23 \\
\hline Principal hospital & 0.24 & Principal hospital ${ }^{* a}$ & 0.21 \\
\hline
\end{tabular}

Note: . Absolute mean index of relative importance (AMI) of factors were displayed in descending order.

${ }^{a}$ Variables with asterisks indicate pProtective effects.

[Correction added on 3 December 2019, after first online publication: In Table 3, 'Sociodemographic status' has been corrected to 'Socioeconomic status' in this current version.]

(Table 2), consistently outperforming the logistic regression model across different severity of co-morbidities. This comparison of prediction models reassures the utility of ANNs in predicting post-operative in-hospital mortality in CRC patients in similar settings with the features of capturing high-order interactions, non-linear relationships, and multicorrelation between input variables. This demonstrates that the current analysis is a better method for informing clinical decision making

We found that in-hospital mortality following acute surgical care in CRC patients was associated with several hospital, clinical, and sociodemographic factors, which is consistent with previous studies reporting poorer post-operative outcomes in association with older age, severity of co-morbidities, rurality of residence, socio-economic disadvantage, and hospital characteristics. ${ }^{8,10,20}$ However, few studies have focused on relative contribution of each variable in relation to post-operative outcomes. The results in this study confirmed some of the findings of previous studies, but with a much larger data set and with a method that achieves higher predictive accuracy by modelling the possibly cumulative and non-linear effects of multiple hospital, clinical, and sociodemographic factors.

The in-hospital mortality during acute surgical care in CRC inpatients was $7.4 \%$ in this study underlining the importance of considering how this could be reduced. Co-morbid musculoskeletal disorders appear to be a key determinant of deaths during acute surgical care among both the co-morbid severity groups. Among our study population with musculoskeletal disorders, approximately $85 \%$ of them had bone mineral density disorders (eg, osteoporosis), which were also reported in other studies as being significant predictors of postoperative mortality in cancer patients. ${ }^{31,32}$ Reduced bone mineral density may be inversely associated with adenomas of the colon, precursors for CRC, and may also be decreased by reduced exercise, reduced levels of vitamin $\mathrm{D}$, and reduced levels of oestrogen. ${ }^{33} \mathrm{In}$ our patient population, patients may have had more advanced stages of CRC, and therefore, their perioperative care was more complicated. For example, chemotherapy might be in place preceding or following surgeries in advanced CRC patients, which could have an adverse impact on musculoskeletal function, ${ }^{34}$ further develop bone metastasis, ${ }^{35}$ impair the immune system, ${ }^{31,36}$ and consequently elevate the post-operative mortality. ${ }^{31,36}$ Given previous findings that bisphosphonates-antiresorptive medications indicated for treating osteoporosis-could reduce the post-operative mortality for CRC, ${ }^{37,38}$ our study reinforces the importance of musculoskeletal health in CRC surgical patients. Osteoporosis is preventable and reversible. ${ }^{39}$ Provision of appropriate interventions such as nutritional support and exercise regimes preceding and following surgeries may improve outcomes. Future clinical trials would clarify the effectiveness of such perioperative musculoskeletal care in CRC patients.

Co-morbid mental disorders appeared to be another important predictor for in-hospital deaths among CRC surgical patients with moderate to severe co-morbidities. Cancer patients with co-morbid depression have been found to have an increased level of interleukin- $6,{ }^{40}$ which is a major cytokine in the tumour microenvironment and an important biomarker highly associated with tumour growth and shorter survival. ${ }^{40,41}$ In addition, cancer patients with co-morbid mental disorders may have a high likelihood of developing cancer metastases and may also delay health care interventions, ${ }^{42}$ resulting in more advanced cancer requiring more aggressive surgeries. Therefore, provision of psychosocial therapy ${ }^{43}$ and/or pharmacological therapy (such as use of interleukin-6 inhibitor) in CRC patients with co-morbid mental disorders during peri-surgical care may provide an avenue to prevent post-operative in-hospital death and improve survival. ${ }^{44}$ Although we were unable to determine the frailty of patients and health service use patterns in the study population, our findings reinforce the impact of co-morbidities on cancer outcomes $^{11}$ and highlight the necessity of identifying appropriate comorbidity care needs in CRC surgical patients. 
Hospital-acquired infections and complications were also major contributors to in-hospital mortalities, which is consistent with previous studies. ${ }^{45-47}$ While strategies for prevention and control of these infections and complications could be informed by surveillance initiatives, ${ }^{48}$ the national surveillance programme is not currently available in Australia. ${ }^{49}$ Recently, the National Safety and Quality Health Service Standards (Australia) highlighted the need for patient safety assurance mechanisms, including appropriate antimicrobial prescribing. ${ }^{50}$ The current findings demonstrate a clear need to reinforce its implementation in acute care settings. Although there was a decreasing trend of in-hospital mortality over time (ie, Table 3, protective effect for fiscal year), continuing efforts such as implementation of surveillance initiatives ${ }^{48,49}$ and provision of qualified perioperative specialist practitioners are encouraged to both reduce hospitalacquired infections and complications and improve survival in surgical patients with CRC. ${ }^{51,52}$

It has been widely reported that poor post-operative outcomes are associated with lower socio-economic status, living in rural areas, older age, nonteaching hospitals, or non-private insured payer status. $^{8,20,53}$ We also observed that their effects on post-operative deaths varied according to different severity of co-morbidity status. For example, having private insurance was associated with elevated mortality risks in CRC patients with less severe co-morbidities but was relatively protective in those with moderate to severe comorbidities. Further investigation is needed to evaluate this finding and identify the potential reasons for this observed contradiction.

In view of the foregoing, our study reinforces the need for the complex peri-surgical decision-making process in CRC patients to include consideration of rurality of residence and socio-economic status as well other more clinically orientated factors if outcomes are to be improved and in-hospital mortality reduced.

Using a complex and flexible non-linear ANN modelling system, this study provides insight into the relative importance of coexisting conditions in relation to post-operative death and how they varied across severity groups, as an evidence-based reference for clinicians to assess and mitigate risk of surgical interventions for CRC patients with complex needs. Although ANN models cannot report risk estimates on individual factors, the higher prediction accuracy in comparison with other conventional generalized linear modelling systems could lead to ANNs becoming a useful tool to enhance understanding of the post-operative in-hospital mortality risks in acute surgical care settings.

There are some limitations in our study. First, the use of administrative APDC data lacks etiological information and so restricts our ability to establish causal associations. We were unable to identify the possible effects of unmeasured clinical and hospital factors such as the aggressiveness of the cancer or the perioperative care guidelines and programmes in relation to in-hospital mortality. Second, individual surgical procedures were not isolated, resulting in the potential for the heterogeneity between procedures to be contributing in part to the observed variation in relative importance of certain co-morbidities. Third, we simplified some clinical variables, eg, dichotomising a condition as present or absent rather than employing complex classification systems to fully appraise their variation. Therefore, the study results should be interpreted with caution. Additionally, our analysis was restricted to public hospitals and was not able to control for the differences between the private and public sectors in relation to postoperative in-hospital mortality. Therefore, the results may not generalize to the private sector. Finally, because age was excluded in calculating the severity of co-morbidities, misclassification for severity group assignment might have occurred. However, we have accounted for the effect of age groups in the ANN modelling for both severity groups, so the impact of such misclassification on our results should have been minimized.

\section{5 | CONCLUSION}

ANN performed better than conventional logistic regression in identifying risk factors for in-hospital mortality in this data set. The findings highlight the variation in relative importance of clinical and sociodemographic factors in association with post-operative inhospital mortality in CRC patients with two different categories of co-morbidity severity. These findings suggested that care plans that incorporate better care strategies for those with co-morbid musculoskeletal and mental health conditions and supportive care elements for those living in disadvantaged conditions would produce better outcomes from CRC surgery. Further investigation is warranted to illuminate the mechanisms underlying these observations.

\section{ACKNOWLEDGEMENTS}

We thank the NSW Health Department for providing the APDC data. The study sponsors had no further role in the study design, data collection, analyses, interpretation of results, writing of the article, or the decision to submit it for publication. We also thank the reviewers for their insightful suggestions for improvement.

\section{CONFLICT OF INTEREST}

The authors declare no conflict of interest

\section{ORCID}

Sha Sha (1) https://orcid.org/0000-0002-7179-611X

Wei Du (D) https://orcid.org/0000-0003-3622-2265

\section{REFERENCES}

1. Arnold M, Sierra M, Laversanne M, Soerjomataram I, Jemal A, Bray F. Global patterns and trends in colorectal cancer incidence and mortality. Gut. 2016;66(4):683-691.

2. Australian Institute of Health and Welfare 2017. Cancer in Australia. Canberra: AlHW: Cancer series no.101. Cat. no. CAN 100; 2017.

3. Wiegering A, Ackermann S, Riegel J, et al. Improved survival of patients with colon cancer detected by screening colonoscopy. Int J Colorectal Dis. 2016;31(5):1039-1045.

4. Cancer Australia. Cancer research in Australia: an overview of funding initiatives to support cancer research capacity in Australia 2006 to 2011. Cancer Australia: Surry Hills, NSW; 2015. 
5. Janssen-Heijnen M, Maas $\mathrm{H}$, Houterman $\mathrm{S}$, Lemmens $\mathrm{V}$, Rutten $\mathrm{H}$, Coebergh J. Comorbidity in older surgical cancer patients: influence on patient care and outcome. Eur J Cancer. 2007;43(15):2179-2193.

6. Alves A. Postoperative mortality and morbidity in french patients undergoing colorectal surgery. Arch Surg. 2005;140(3):278. 83, discussion 284

7. Lemmens $V$, Janssen-Heijnen M, Houterman S, et al. Which comorbid conditions predict complications after surgery for colorectal cancer? World J Surg. 2006;31(1):192-199.

8. Beckmann K, Moore J, Wattchow D, Young G, Roder D. Short-term outcomes after surgical resection for colorectal cancer in South Australia. J Eval Clin Pract. 2016;23(2):316-324.

9. Usher-Smith J, Miller R, Griffin S. Predicting survival in patients with colorectal cancer. BMJ. 2017;357:j2772.

10. Iversen LH, Nørgaard M, Jacobsen J, Soren L, Sørensen HT. The impact of comorbidity on survival of Danish colorectal cancer patients from 1995 to 2006-a population-based cohort study. Dis Colon Rectum. 2009;52(1):71-78.

11. Søgaard M, Thomsen RW, Bossen KS, Sørensen HT, Nørgaard M. The impact of comorbidity on cancer survival: a review. Clin Epidemiol. 2013;5(Suppl 1):3-29.

12. Geraci JM, Escalante CP, Freeman JL, Goodwin JS. Comorbid disease and cancer: the need for more relevant conceptual models in health services research. J Clin Oncol Official J American Soc Clin Oncol. 2005;23(30):7399-7404.

13. Dormann C, Elith J, Bacher S, et al. Collinearity: a review of methods to deal with it and a simulation study evaluating their performance. Ecography. 2012;36(1):27-46.

14. Dreiseitl S, Ohno-Machado L. Logistic regression and artificial neural network classification models: a methodology review. J Biomed Inform. 2002;35(5-6):352-359.

15. Bottaci L, Drew P, Hartley J, et al. Artificial neural networks applied to outcome prediction for colorectal cancer patients in separate institutions. The Lancet. 1997;350(9076):469-472.

16. Hall S, Holman C, Platell C, Sheiner H, Threlfall T, Semmens J. Colorectal cancer surgical care and survival: do private health insurance, socioeconomic and locational status make a difference? ANZ J Surg. 2005;75(11):929-935

17. Jackson TJ, Michel JL, Roberts RF, Jorm CM, Wakefield JG. A classification of hospital-acquired diagnoses for use with routine hospital data. Med J Aust. 2009;191(10):544-548.

18. Australian Bureau of Statistics (ABS). Information Paper 2039.0: an introduction to socio-economic indexes for areas (SEIFA). Canberra: ABS; 2006.

19. Australian Institute of Health and Welfare. Australian hospital statistics 2012-13. Health services series no. 54. Cat. no. HSE 145. Canberra: AlHW; 2014.

20. van Groningen J, Eddes E, Fabry H, et al. Hospital teaching status and patients' outcomes after colon cancer surgery. World J Surg. 2018;42(10):3372-3380.

21. Borzecki A, Christiansen C, Chew P, Loveland S, Rosen AK. Comparison of in-hospital versus 30-day mortality assessments for selected medical conditions. Med Care. 2010;48(12):1117-1121.

22. Ben-Tovim D, Woodman R, Harrison J, et al. Measuring and reporting mortality in hospital patients (Cat: HSE 69). Canberra: AlHW; 2009.

23. Olden JD, Jackson DA. Illuminating the "black box": a randomization approach for understanding variable contributions in artificial neural networks. Ecol Model. 2002;154(1):135-150.
24. Olden JD, Joy MK, Death RG. An accurate comparison of methods for quantifying variable importance in artificial neural networks using simulated data. Ecol Model. 2004;178(3):389-397.

25. Charlson ME, Pompei P, Ales KL, et al. A new method of classifying prognostic comorbidity in longitudinal studies: development and validation, J Chronic Dis, 1987, vol. 40 5(pg. 373-383)

26. Quan H, Li B, Couris C, et al. Updating and validating the Charlson comorbidity index and score for risk adjustment in hospital discharge abstracts using data from 6 countries. Am J Epidemiol. 2011;173(6):676-682.

27. Brunelli A, Postmus PE. Preoperative functional evaluation of the surgical candidate. Second ed. 2018;265-73.e3.

28. De Oña J, Garrido C. Extracting the contribution of independent variables in neural network models: a new approach to handle instability. Neural Comput Applic. 2014;25(3-4):859-869.

29. Practical guide to deal with imbalanced classification problems in $\mathrm{R}$ [Internet]. Analytics Vidhya. 2019. Available at: https://www. analyticsvidhya.com/blog/2016/03/practical-guide-deal-imbalancedclassification-problems/. Accessed at: 1 March 2019.

30. Arnold T. Uninformative parameters and model selection using Akaike's information criterion. J Wildlife Manage. 2010;74(6):1175-1178.

31. Miyachi Y, Kaido T, Yao S, et al. Bone mineral density as a risk factor for patients undergoing surgery for hepatocellular carcinoma. World $\mathrm{J}$ Surg. 2018;43(3):920-928.

32. Drake M. Osteoporosis and cancer. Curr Osteoporos Rep. 2013;11(3):163-170.

33. Nock N, Patrick-Melin A, Cook M, Thompson C, Kirwan J, Li L. Higher bone mineral density is associated with a decreased risk of colorectal adenomas. Int J Cancer. 2010;129(4):956-964.

34. Safaei-Nodehi R, Esmaili J, Sharifian R, Movaseghi S, Parkhideh S. Does adjuvant chemotherapy change bone mineral density and related serum biomarkers in women with breast cancer? Caspian J Intern Med. 2017;8(2):91-98.

35. Assi R, Mukherji D, Haydar A, Saroufim M, Temraz S, Shamseddine A. Metastatic colorectal cancer presenting with bone marrow metastasis: a case series and review of literature. J Gastroin Oncol. 2016;7(2):284.

36. Li H, Hong S, Qian J, Zheng Y, Yang J, Yi Q. Cross talk between the bone and immune systems: osteoclasts function as antigenpresenting cells and activate CD4+ and CD8+ T cells. Blood. 2010;116(2):210-217.

37. Cardwell C, Abnet C, Veal P, Hughes C, Cantwell M, Murray L. Exposure to oral bisphosphonates and risk of cancer. Int J Cancer. 2012;131(5):E717-E725.

38. Pazianas M, Abrahamsen B, Eiken P, Eastell R, Russell R. Reduced colon cancer incidence and mortality in postmenopausal women treated with an oral bisphosphonate-Danish National Register Based Cohort Study. Osteoporos Int. 2012;23(11):2693-2701.

39. Lustberg M, Reinbolt R, Shapiro C. Bone health in adult cancer survivorship. J Clin Oncol. 2012;30(30):3665-3674.

40. Jehn C, Kuehnhardt D, Bartholomae A, et al. Biomarkers of depression in cancer patients. Cancer. 2006;107(11):2723-2729.

41. Kumari N, Dwarakanath B, Das A, Bhatt A. Role of interleukin-6 in cancer progression and therapeutic resistance. Tumor Biology. 2016;37(9):11553-11572.

42. Kisely S, Crowe E, Lawrence D. Cancer-related mortality in people with mental illness. JAMA Psychiat. 2013;70(2):209-217. 
43. Nakash O, Levav I, Aguilar-Gaxiola S, et al. Comorbidity of common mental disorders with cancer and their treatment gap: findings from the World Mental Health Surveys. Psychooncology. 2013;23(1):40-51.

44. Hodes G, Ménard C, Russo S. Integrating Interleukin-6 into depression diagnosis and treatment. Neurobiol Stress. 2016;4:15-22.

45. Klevens R, Edwards J, Richards C, et al. Estimating health careassociated infections and deaths in U.S. hospitals, 2002. Public Health Rep. 2007;122(2):160-166.

46. Astagneau P, Rioux C, Golliot F, Brücker G. Morbidity and mortality associated with surgical site infections: results from the 1997-1999 INCISO surveillance. J Hosp Infect. 2001;48(4):267-274.

47. Guzman-Pruneda F, Husain S, Jones C, et al. Compliance with preoperative care measures reduces surgical site infection after colorectal operation. J Surg Oncol. 2018;119(4):497-502.

48. British Medical Association. Tackling healthcare associated infections through effective policy action. London: BMA; 2009.

49. Russo P, Cheng A, Mitchell B, Hall L. Healthcare-associated infections in Australia: tackling the 'known unknowns'. Aust Health Rev. 2018;42(2):178.

50. Australian Commission on Safety and Quality in Health Care. National Safety and Quality Health Service Standards. ACSQHC, Sydney, 2011.
Available at https://www.safetyandquality.gov.au/wp-content/ uploads/2011/01/NSQHS-Standards-Sept2011.pdf. Accessed at: 12 Dec, 2018.

51. Story D. Postoperative complications in Australia and New Zealand (the REASON study). Perioperative Medicine. 2013;2(1):16.

52. Kneebone R, Nestel D, Chrzanowska J, et al. The perioperative specialist practitioner: developing and evaluating a new surgical role. Qual Saf Health Care. 2006;15(5):354-358.

53. Xu H, White R, Sastow D, Andreae M, Gaber-Baylis L, Turnbull Z. Medicaid insurance as primary payer predicts increased mortality after total hip replacement in the state inpatient databases of California, Florida and New York. J Clin Anesth. 2017;43:24-32.

How to cite this article: Sha S, Du W, Parkinson A, Glasgow N. Relative importance of clinical and sociodemographic factors in association with post-operative in-hospital deaths in colorectal cancer patients in New South Wales: An artificial neural network approach. J Eval Clin Pract. 2019. https://doi. org/10.1111/jep.13318 